\title{
ESTIMATIVA DAS ESTABILIDADES LONGITUDINAL E TRANSVERSAL DE TRATORES FLORESTAIS UTILIZADOS NA COLHEITA DE MADEIRA ${ }^{1}$
}

\author{
Julião Soares de Souza Lima², Amaury Paulo de Souza ${ }^{3}$, Carlos Cardoso Machado³ , José Eduardo \\ Macedo Pezzopane ${ }^{2}$ e Mário Luis Areas ${ }^{4}$
}

\begin{abstract}
RESUMO - Este trabalho teve como objetivo estimar as estabilidades longitudinal e transversal nas condições dinâmicas de tráfego, com base na estabilidade estática e nas coordenadas do centro de gravidade (CG) de dois tratores com rodados de pneus, sendo um derrubador-amontoador ("Feller-buncher") e outro arrastador ("Skidder"), utilizados em um sistema de aproveitamento de toras compridas em povoamento de eucalipto. O resultado do trator "Skidder" permitiu seu tráfego transversal em rampas com inclinação de no máximo 33,2\%. No caso do "Feller-buncher", seu uso deve ser recomendado sempre em terrenos planos, em virtude de suas características técnicas e operacionais, com grande concentração de carga no eixo dianteiro quando deslocando carregado.
\end{abstract}

Palavras-chave: Estabilidade, centro de gravidade e tratores florestais.

\section{LONGITUDINAL AND TRANSVERSAL STABILITY ESTIMATE FOR THE TRAFFIC OF FOREST TRACTORS USED IN WOOD HARVESTING}

\begin{abstract}
The objective of this work was to estimate the longitudinal and transversal stability for dynamic traffic conditions, based on the coordinates of the gravity center $(C G)$ of two tractors, a Feller-buncher and a Skidder, equipped with driving wheels of tires, used in a long logs utilization system in a eucalyptus stand. The tractor Skidder presented a value of inclination of transversal ramp of up to 33,2\%. As for the "Fellerbuncher", its use should always be recommended on plain lands, because of its technical and operational characteristics, with great load concentration in the front axis when moving loaded.
\end{abstract}

Key Words: Stability, center of gravity, forest tractors.

\section{INTRODUÇÃO}

No Brasil, a colheita florestal é muito diversificada, variando segundo cada região geográfica. Hoje, podemse encontrar florestas plantadas em áreas muito íngremes, bem como planas. Esse fato leva à adoção pelas empresas florestais de diferentes técnicas mecanizadas na etapa da colheita de madeira.

A realização de algumas atividades do setor florestal pode ser limitada em regiões de topografia acidentada, por não permitirem o tráfego das máquinas com estabilidade. A estabilidade, longitudinal e transversal, está relacionada com a capacidade de uma máquina subir, descer e trafegar lateralmente em curva de nível sem comprometer sua dirigibilidade.

A introdução de máquinas para a realização de tarefas que antes eram realizadas manualmente motivou,

\footnotetext{
${ }^{1}$ Recebido em 25.02.2003 e aceito para publicação em 10.08.2004.

${ }^{2}$ Departamento de Engenharia Rural da UFES, Alegre, ES. Cx. Postal 16, CEP 29500-000. E-mail: <juliaosslima@cca.ufes.br>.

${ }^{3}$ Departamento de Engenharia Florestal da UFV, Viçosa, MG. CEP 36570-000. E-mail: <machado@ ufv.br>.

${ }^{4}$ Agrônomo.
} 
em parte, a organização do trabalho. Entretanto, expõem os operadores a riscos de acidentes provocados pela falta de condições mínimas de segurança. Essa situação se agrava ainda mais quando são constatados casos em que trabalhadores despreparados e, portanto, sem um treinamento adequado propõem-se a operar máquinas sofisticadas, podendo contribuir para a ocorrência de acidentes de trabalho, em alguns casos com extrema gravidade.

No início, os projetos dos tratores estavam mais voltados para a utilização e redução dos custos de produção do equipamento. Com o desenvolvimento tecnológico, passou-se a buscar o aperfeiçoamento da relação homem-máquina, com a finalidade de impor ao homem uma carga de trabalho mais suave, visando à redução da fadiga e, em conseqüência, ao aumento da produtividade do conjunto e à melhoria do serviço executado (MENEZEZ et al., 1985).

Algumas máquinas podem operar satisfatoriamente em terrenos acidentados sem comprometer a dirigibilidade e segurança do operador. Entretanto, esse fato não minimiza os efeitos causados à sua postura no posto de trabalho, proporcionando desconforto e podendo levar à ocorrência de problemas na coluna do operador em decorrência da movimentação das máquinas sobre superfícies irregulares (LIMA, 1998).

Estima-se que de $85 \%$ dos acidentes fatais, em que há o envolvimento de tratores agrícolas, $70 \%$ são devidos a quedas laterais e $15 \%$ ao tombamento para trás (FUNDACENTRO, 1979). Robin (1987) afirmou que a maior ocorrência de óbitos no meio rural está diretamente relacionada com as operações de tratores agrícolas em condições não-favoráveis ao tráfego.

Considerando a gravidade dos acidentes de trabalho, segundo Delgado (1991), cerca de 40\% dos que ocorrem no setor agrário na Espanha são causados por máquinas agrícolas, estando a metade deles relacionada a operações com tratores.

Para Mialhe (1980), o estudo das forças que agem sobre o chassi do trator tem fundamental importância para a compreensão de certos fenômenos que ocorrem durante sua utilização em campo. O conhecimento das condições de equilíbrio permite a utilização mais segura do trator, evitando-se riscos de acidentes, principalmente em terrenos de topografia acidentada.

Khouri Jr. (2000) realizou estudo da estabilidade de tratores agrícolas em condições desfavoráveis ao tráfego, considerando-se que o trator está apoiado sobre três pontos, ou seja, nos dois rodados traseiros e no mancal que une o chassi ao eixo frontal, utilizando para tanto o método da dupla pesagem para a determinação do seu centro de gravidade.

Quanto maior for a velocidade de deslocamento, mais intensamente se manifesta a ação dos processos dinâmicos, que podem provocar o tombamento lateral de uma máquina. Devido a esse fato, recomenda-se que o ângulo do terreno que permite a estabilidade dinâmica durante a realização de uma operação deve estar entre 40 e $60 \%$ do ângulo-limite máximo determinado em condição estática (CHUDAKOV, 1977; MIALHE, 1980).

O objetivo deste trabalho é estimar a declividade do terreno que permite o tráfego com estabilidade longitudinal e transversal, em razão dos dados dimensionais e ponderais e das coordenadas do centro de gravidade de dois tratores florestais utilizados em colheita de madeira.

\section{MATERIAL E MÉTODOS}

Este trabalho foi realizado em tratores florestais utilizados em um sistema de aproveitamento das árvores em toras compridas. As etapas da colheita foram realizadas pelos seguintes tratores:

Corte: trator florestal derrubador-amontoador ("Feller-Buncher"), com cabine de proteção, tração tipo $4 \times 4$, pneus 23.1-26, pressão interna de inflagem de $193 \mathrm{kPa}$, raio estático dos pneus de $790 \mathrm{~mm}$, distância entre os eixos de $2.540 \mathrm{~mm}$, bitola de $2.000 \mathrm{~mm}$, distribuição de peso no eixo dianteiro de 53,9\%, massa total de $13.375 \mathrm{~kg}$, motor Cummins 6BT 5.9, potência de $105 \mathrm{~kW}, 6$ cilindros, relação peso/potência de 127,4 $\mathrm{kg} / \mathrm{kW}$ e montado com cabeçote frontal com disco dentado para o corte.

Arraste: trator florestal arrastador ("Skidder"), articulado, tração tipo 4x4, motor CAT 3304 DIT, potência de $130 \mathrm{~kW}, 4$ cilindros, com cabine de proteção, pneus (BPAF) 66 x 43-26 “, raio estático dos pneus de 850 $\mathrm{mm}$, pressão interna de inflagem de $179 \mathrm{kPa}$, distância entre eixos de $3.500 \mathrm{~mm}$, bitola de $2.700 \mathrm{~mm}$, distribuição de peso no eixo dianteiro de $55 \%$, massa total de 16.245 $\mathrm{kg}$, relação peso/potência de $125 \mathrm{~kg} / \mathrm{kW}$, montado com torre hidráulica de dupla ação para o abraçamento das bandeiras de toras, com uma área de 1,25 m²". 
Para o estudo da estabilidade das máquinas é necessário conhecer as características dimensionais e ponderais para a determinação das coordenadas do centro de gravidade (CG), que definem o ponto geométrico no corpo da máquina onde a força peso total está atuando normal à superfície do solo. Essas coordenadas são definidas em três planos ortogonais, a saber: plano de apoio, plano médio longitudinal e plano transversal traseiro, conforme ilustrado na Figura 1 AB O método utilizado é o da "dupla pesagem". Determinando a distribuição da massa do corpo para as laterais e sobre os eixos frontal e traseiro da máquina, considerou-se o trator como um corpo rígido com quatro pontos de apoio sobre o solo (CHUDAKOV, 1977).

A coordenada longitudinal horizontal do trator (X) está contida no plano longitudinal do trator a partir do plano transversal que corta o eixo traseiro, sendo calculada pela seguinte equação:

$$
\mathrm{X}=\frac{\mathrm{R} 2 * \mathrm{DE}}{\mathrm{W}}
$$

em que:

$\mathrm{X}=$ coordenada longitudinal $(\mathrm{mm})$;

$\mathrm{W}=$ massa total do trator $(\mathrm{kg})$;

$\mathrm{R} 2$ = reação do solo contra as rodas dianteiras, indicada por uma balança $(\mathrm{kg})$; e

$\mathrm{DE}=$ distância entre o centro dos eixos dianteiro e traseiro $(\mathrm{mm})$.

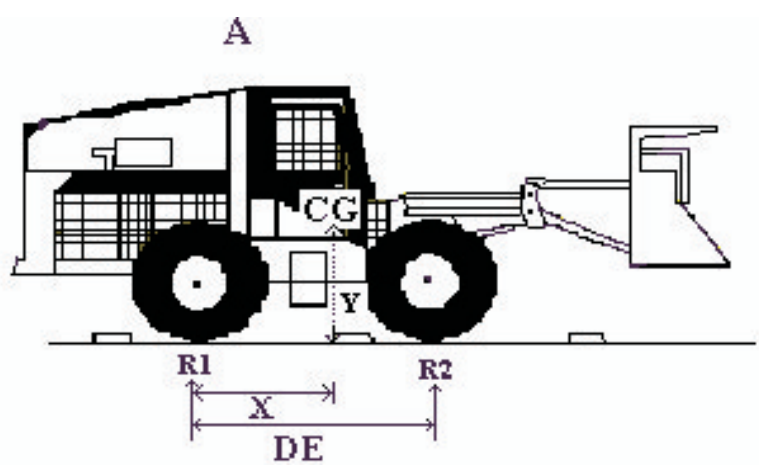

A coordenada horizontal traseira (Z) está contida no plano transversal que passa pelo centro do eixo traseiro, sendo determinada pela equação 2 .

$$
\mathrm{Z}=\mathrm{a} * \frac{(\mathrm{R} 4-\mathrm{R} 3)}{2 * \mathrm{~W}}
$$

em que:

$$
\begin{aligned}
& \mathrm{Z}=\text { coordenada horizontal traseira }(\mathrm{mm}) \\
& \mathrm{a}=\text { bitola do trator }(\mathrm{mm})
\end{aligned}
$$

$\mathrm{R} 4$ = reação do solo contra o lado direito do trator, indicada por uma balança $(\mathrm{kg})$;

R3 = reação do solo contra o lado esquerdo do trator $(\mathrm{kg})$, indicado por uma balança; e

$$
\mathrm{W}=\text { massa total do trator }(\mathrm{kg}) \text {. }
$$

A coordenada vertical (Y) é a distância do CG ao plano horizontal de apoio, tomada na perpendicular baixada pelo CG a esse plano. Para a determinação da coordenada vertical, eleva-se o eixo dianteiro sobre determinada balança de plataforma a certa altura de forma a atingir um ângulo de aproximadamente $15^{\circ}$, conforme descrito por Chudakov (1977). Essa coordenada é determinada pela seguinte equação:

$$
\mathrm{Y}=\mathrm{r} 1+\left(\mathrm{X}-\frac{\mathrm{X} 1}{\cos \beta 1}\right) * \operatorname{ctg} \beta 1
$$

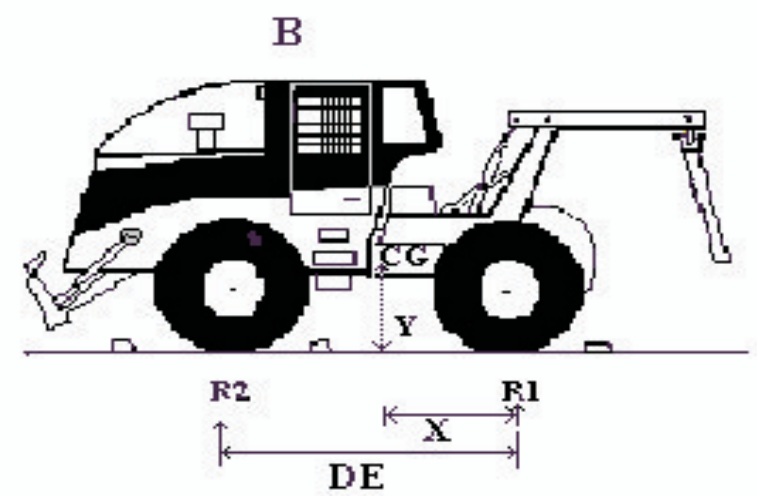

Figura 1 - Representação das coordenadas do CG no trator "Feller-buncher" (A) e no trator "Skidder" (B). Figure 1 -Representation of CG coordinates in the Feller-buncher (A) and in the Skidder (B). 
em que:

$$
\begin{aligned}
& \mathrm{Y}=\text { coordenada vertical do } \mathrm{CG}(\mathrm{mm}) \\
& \mathrm{r} 1 \text { = raio estático do rodado traseiro }(\mathrm{mm}) ; \\
& \mathrm{X} 1 \text { = projeção da coordenada horizontal longitudinal }
\end{aligned}
$$
(X) no plano de apoio, quando o eixo dianteiro estiver elevado ( $\mathrm{mm})$; e

$\beta 1$ = ângulo de inclinação do eixo dianteiro (graus).

Com certa aproximação, pode-se considerar que:

$$
\beta 1=\operatorname{arctg}\left(\frac{\mathrm{K}}{\mathrm{DEE}}\right)
$$

em que:

$\mathrm{K}=$ altura da elevação do eixo dianteiro ( $\mathrm{mm})$; e

$\mathrm{DEE}=$ projeção horizontal da distância entre eixos, quando elevado $(\mathrm{mm})$.

Conhecendo as coordenadas do centro de gravidade (CG) dos tratores, determinaram-se as estabilidades longitudinal e transversal em condição estática. A partir desses dados, estimou-se a estabilidade para condição dinâmica de tráfego, em virtude da dificuldade de se determinar as condições de campo, devido alguns fatores, como: tipo de superfície, capacidade de sustentação do solo, carregamento, tipo de rodado, pressão de inflagem etc.

Denominou-se como ângulo-limite máximo de subida de rampa (a) aquele em que o trator se posiciona parado sem deslizar. Para a condição de equilíbrio estático do trator, tem-se:

$$
\alpha=\operatorname{arctg}\left(\frac{X}{Y}\right)
$$

em que:

$\mathrm{a}=$ ângulo-limite de subida de rampa (graus);

$\mathrm{X}=$ coordenada longitudinal horizontal do CG (mm); e

$\mathrm{Y}=$ coordenada vertical do $\mathrm{CG}(\mathrm{mm})$.

O ângulo-limite máximo de descida de rampa $(\theta)$ foi definido com o trator estando posicionado sem deslizar e na condição de descida. Na situação de equilíbrio estático do trator, tem-se:

R. Árvore, Viçosa-MG, v.28, n.6, p.839-844, 2004
$\theta=\operatorname{arctg}\left(\frac{\mathrm{DE}-\mathrm{X}}{\mathrm{Y}}\right)$

em que:

$\theta$ = ângulo-limite máximo de descida (graus);

$\mathrm{DE}=$ distância entre eixos $(\mathrm{mm})$;

$\mathrm{X}=$ coordenada longitudinal horizontal do $\mathrm{CG}(\mathrm{mm})$; e

$\mathrm{Y}=$ coordenada vertical do $\mathrm{CG}(\mathrm{mm})$.

Denominou-se o ângulo-limite máximo de inclinação transversal (g) o ângulo no qual o trator está parado, sem deslizar lateralmente. O valor de g pode ser determinado considerando-se que o tombamento começará quando a reação normal do solo nas rodas laterais dispostas na parte superior da rampa se reduzir a zero. Escrevendo a equação dos momentos em torno de um ponto do pneu com o solo na parte inferior da rampa, tem-se:

$$
\gamma=\operatorname{arctg}\left(\frac{0,50 * \mathrm{a}}{\mathrm{Y}}\right)
$$

em que:

eq. 7

$\gamma=$ ângulo-limite para o tráfego lateral (graus);

$\mathrm{a}=$ bitola do trator $(\mathrm{mm})$; e

$\mathrm{Y}=$ coordenada vertical do $\mathrm{CG}(\mathrm{mm})$.

Para condição dinâmica de tráfego lateral, considerou-se como sendo de $40 \%$ do valor determinado em condição estática, como descreveu Chudakov (1977).

\section{RESULTADOS E DISCUSSÃO}

As coordenadas do centro de gravidade (CG) dos tratores florestais determinadas pelo método da "dupla pesagem" e utilizadas para estimar as estabilidades longitudinal e transversal estão no Quadro 1.

Quadro 1 - Coordenadas do centro de gravidade (CG) dos tratores florestais "Feller-buncher" e "Skidder"

Table 1 -Gravity center coordinates (CG) of the forest tractors "Feller-buncher" and "Skidder"

\begin{tabular}{lccc}
\hline Tratores & $\mathrm{X}(\mathrm{mm})$ & $\mathrm{Y}(\mathrm{mm})$ & $\mathrm{Z}(\mathrm{mm})$ \\
\hline Skidder & 1.963 & 1.310 & 44 \\
Feller-Buncher & 1.370 & 1.210 & 0 \\
\hline
\end{tabular}

$\mathrm{X}=$ coordenada longitudinal horizontal, $\mathrm{Y}=$ coordenada vertical e $\mathrm{Z}=$ coordenada horizontal traseira. 
As coordenadas do CG variam de posição para cada um dos tratores. Isso ocorre em virtude de a massa total e de a distribuição de peso nos tratores serem diferentes. Conhecidas essas coordenadas, estimouse as declividades do terreno para condição de tráfego dinâmico.

Os ângulos-limite de subida de rampa $(\alpha)$ estimados para os tratores "Skidder" e "Feller-buncher" foram de $56,3^{\circ}(149,8 \%)$ e $48,5^{\circ}(113,2 \%)$, respectivamente, enquanto os ângulos-limite de descida de rampa $(\theta)$ estimados para os tratores "Skidder" e "Feller-buncher" foram de $49,6^{\circ}(117,3 \%)$ e $44,0^{\circ}(96,7 \%)$, respectivamente.

Os valores estimados estão maiores do que os previstos por Chudakov (1977), que estão na faixa de $35^{\circ}(70,0 \%)$ a $40^{\circ}(83,9 \%)$. Esse fato está relacionado com a distribuição estática de peso nos dois tratores, que é de 55\% no eixo frontal para o "Skidder" e 53,9\% para o "Feller-buncher".

A estabilidade transversal, estimada para o ângulo $\gamma$ na condição dinâmica de tráfego, foi considerada nos dois tratores como sendo $40 \%$ do valor do ângulo obtido na condição estática. Para o "Feller-buncher" foi determinada uma inclinação-limite de $28 \%$ e para o "Skidder", de 33,2\%. A montagem do "Skidder" com pneus de baixa pressão e alta flutuação (BPAF) confere maior estabilidade transversal que o "Feller-buncher". O valor da inclinação estimada na condição de tráfego dinâmico para o “Skidder"está próximo dos 35\%, conforme Valverde (1995).

A operação realizada pelo "Feller-buncher" proporciona maior instabilidade transversal em função do: peso, altura e ação do vento sobre as copas das árvores; subida em saliências na área de corte, como cepas; pouca capacidade de sustentação do solo; abaixamento da pressão interna de inflagem dos pneus; habilidade do operador; etc. Em razão desses fatores, deve-se recomendar, com restrição, o tráfego do trator carregado transversalmente à inclinação do terreno, apesar de o valor estimado experimentalmente recomendá-lo.

Não deve ser recomendado o uso do trator "Fellerbuncher" em terrenos com inclinação estimada para o ângulo-limite de descida de rampa $(\theta)$, em virtude de suas características técnicas e operacionais, com grande concentração de carga no eixo dianteiro quando carregado com árvores, podendo levar a acidentes.
Com o conhecimento das inclinações-limite para o tráfego e com um modelo digital de elevação de uma região, é possível determinar a potencialidade do uso de máquinas e equipamentos por determinada empresa na colheita de madeira.

\section{CONCLUSÕES}

Diante dos resultados, chegou-se às seguintes conclusões:

- A metodologia utilizada permite estimar, com certa precisão, a inclinação do terreno para tráfegos longitudinal e transversal, recomendada para operação dos referidos tratores.

- Maior distribuição de peso dos referidos tratores no eixo dianteiro facilita a subida em rampas íngremes. Os ângulos estimados para os tratores "Skidder" e "Feller-Buncher" foram de 56,3 e 48,5 $5^{\circ}$, respectivamente.

- Em virtude das características técnicas e operacionais do trator "Feler-buncher", com grande concentração de carga no eixo dianteiro quando carregado com árvores, não deve ser recomendado o seu uso em terrenos com a inclinação estimada para o ângulo -limite de descida de rampa ( q ) encontrado.

- Quanto maior for a bitola dos tratores e mais próximo do solo estiver a coordenada vertical (Y) do CG, com mais segurança pode-se trafegar transversalmente em terrenos inclinados.

\section{REFERÊNCIAS BIBLIOGRÁFICAS}

CHUDAKOV, D. A. Fundamentos de la teoria y el cálculo de tractores y automóviles. Moscow: Mir, 1977.435p.

DELGADO, L. M. El tractor agrícola características y utilizacion. Madrid: Laboreo Solotractor, 1991. 235p.

FUNDACENTRO - Manual de segurança, higiene e medicina do trabalho rural. São Paulo: 1979. 84 p.

KHOURY JR, J. K. Estabilidade lateral de tratores agrícolas 4x2. 2000. $95 \mathrm{f}$.

Dissertação (Mestrado em Engenharia Agrícola). Universidade Federal de Viçosa, Viçosa, 2000.

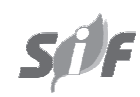

R. Árvore, Viçosa-MG, v.28, n.6, p.839-844, 2004 
LIMA, J. S. S. Avaliação da força de arraste, compactação do solo e fatores ergonômicos num sistema de colheita de madeira utilizando os tratores "Feller-buncher" e "Skidder". 1998.128f. Tese (Doutorado em Ciência Florestal) Universidade Federal de Viçosa, Viçosa, 1998.

MENEZEZ, J. F. et al. Avaliação de características de visibilidade apresentada por um grupo de tratores de rodas. Campinas: Instituto Agronômico de Campinas, 1985. 18p.
MIALHE, L. G. Máquinas motoras na agricultura. São Paulo: EDUSP, 1980. v.2, 367p.

ROBIN, P. Segurança e ergonomia em máquinas agrícolas. São Paulo: IPT, FUNDACENTRO, 1987.24p.

VALVERDE, S. R. Análise técnica e econômica do sistema de colheita de árvores inteiras em povoamentos de eucalipto. 1995. 123f. Dissertação(Mestrado em Ciência Florestal)- Universidade Federal de Viçosa, Viçosa, 1995. 